\title{
T cells are depleted in HCV-induced hepatocellular carcinoma patients: possible role of apoptosis and p53
}

\author{
Sherif A Ibrahim ${ }^{1}$, Sayed F Abdelwahab*2, Mona M Mohamed ${ }^{1}$, \\ Ahmed M Osman ${ }^{1}$, Eman Fathy ${ }^{3}$, Kamal S Al-Badry ${ }^{1}$, Nabil Al-Kady ${ }^{4}$, \\ Gamal Esmat ${ }^{4}$ and Maged M Al-Sherbiny ${ }^{1}$
}

\author{
Address: ${ }^{1}$ Department of Zoology, Faculty of Science, Cairo University, Giza, Egypt, ${ }^{2}$ Microbiology and Immunology Faculty of Medicine, Minia \\ University, Minia, Egypt, ${ }^{3}$ VacSera, Giza, Egypt and ${ }^{4}$ Tropical Medicine, Faculty of Medicine, Cairo University, Giza, Egypt \\ * Corresponding author
}

from 2006 International Meeting of The Institute of Human Virology Baltimore, USA. 17-21 November, 2006

Published: 21 December 2006

Retrovirology 2006, 3(Suppl I):S32 doi:I0.II86/I742-4690-3-SI-S32

(c) 2006 Ibrahim et al; licensee BioMed Central Ltd.

Egypt has possibly the highest Hepatitis C Virus (HCV) prevalence worldwide. A high proportion of HCV infections become chronic and lead to liver cirrhosis and hepatocellular carcinoma (HCC). The cellular and molecular mechanisms behind the complication of HCV infection are not completely understood although apoptosis has been implicated in this process. We examined whether $\mathrm{T}$ cells; isolated from patients with HCV and HCV-associated HCC (HCV-HCC); are predestined in vivo to undergo spontaneous apoptosis using flow cytometry. Also, the role of p53; a key in apoptotic process; in the development of HCC was examined. Our data showed that T cells were severely depleted in HCV-HCC patients and its spontaneous apoptosis was higher in patient groups as compared to normal controls as did the expression of p53, which correlated well with the HCC grade. In conclusion, HCV infection induces peripheral T cell apoptosis, depletion and subsequently immune-suppression in HCV patients and this may lead to persistence of infection. Also, p53 is implicated in the poor prognosis of HCV-HCC and could be used as a biomarker to assess the prognosis of HCC patients. 